\title{
Shape Controlled Palladium Nano Particles for Hydrogenation of Cinnamaldehyde
}

\author{
Surya Kumar Vatti, Krishnamurthy Konda Ramaswamy, Viswanathan Balasubramanaian* \\ National Centre for Catalysis Research (NCCR) Indian Institute of Technology Madras, Chennai-600036, India \\ Email: bvnathan@iitm.ac.in
}

\begin{abstract}
Palladium nano particles in tetrahedral (Td), octahedral (Oh) and spherical (Sp) shapes, supported on hydrotalcite, were synthesized and characterized by XRD and TEM. Td shaped Pd nano particles, with maximum number of exposed (111) planes of $\mathrm{Pd}$, display maximum turn over frequency (TOF) / intrinsic activity for hydrogenation of cinnamaldehyde, indicating the importance of active site geometry. Oh \& Sp shaped Pd nano particles that expose both (111) and (100) planes have relatively lower intrinsic activity. For a given reaction, it is essential that appropriate active site geometry, as dictated by the shape/morphology of nano particles be made available. Synthesis and stabilization of such shape controlled nano particles is of prime importance in developing catalysts with higher activity.
\end{abstract}

Keywords: Pd nano particles, shape control, active site geometry, cinnamaldehyde, hydrogenation, TOF.

\section{Introduction}

Supported metal catalysts have been extensively studied, as they display high activity, selectivity, stability under mild reaction conditions as required in many catalytic reactions [1]. A number of factors are known to influence the performance of supported metal catalysts [2], namely, the metal loading, nature of metals, crystallite size, metal surface morphology, nature of the support, addition of second/promoter elements [3], the metal-support interactions and method of preparation [4]. Of these, the crystallite size and morphology of the metal particles are the two important factors affecting the number and nature/geometry of the active sites, which in turn influence activity and selectivity respectively. Nano size metal particles with different sizes and shapes have different degrees of dispersion, exposed facets and fractions of atoms located at different corners, edges, and defects, each having specific activity/selectivity. Thus, for a given reaction, the catalytic activity of particles with different morphology can vary significantly. Semagina and Kiwi-Minsker [5] compared the activity of nano size Pd hexagons and spheres for hydrogenation of 2-methyl-3-butyn-2-ol and observed that nano spheres with maximum amount of exposed (111) planes displayed highest TOF. Role of shaped Pd nano crystals in controlling activity \& selectivity for various reactions by influencing the facets, and relative populations of atoms in edges, corners and planes has been reviewed in detail by Zhang et al [6]. Wang et al. [7] reported that silica supported Pd nano particles in octahedral shape (with predominantly (111) planes) was more active for CO oxidation compared to cubic Pd (with (100) facet) while the facet activity pattern was exactly reverse in the case of silica supported Pt. Hence, studies on the preparation, characterization and performance evaluation of shape controlled supported metal catalysts has been area of tremendous importance both from basic as well as applied aspects of catalysis. In the present work, we have explored the effect of Pd nano particles in tetrahedral (Td), octahedral (Oh) and spherical (Sp) shapes, supported on hydrotalcite (HT), using hydrogenation of cinnamaldehyde as the model reaction.

\section{$2 \quad$ Experimental}

\subsection{Materials}

Palladium chloride (Sisco Research Laboratories), poly vinyl pyrrolidone (PVP), ethylene glycol (EG), sodium hydroxide $(\mathrm{NaOH})$, magnesium nitrate hexa hydrate $\left(\mathrm{Mg}\left(\mathrm{NO}_{3}\right)_{2} \bullet 6 \mathrm{H}_{2} \mathrm{O}\right)$, aluminum nitrate 
nonahydrate $\left(\mathrm{Al}\left(\mathrm{NO}_{3}\right)_{3} \bullet 9 \mathrm{H}_{2} \mathrm{O}\right)$, cetyl trimethyl ammonium bromide (CTAB) from Sigma Aldrich, hexamethylenetetramine (HMTA), cinnamaldehyde and methanol (Qualigens) were of analytical reagent (AR) grade and used as such.

\subsection{Preparation of Shape Controlled Pd/Hydrotalcite (HT) Catalysts}

Palladium supported on hydrotalcite catalysts were prepared by adopting precipitation-reduction method [8] wherein the precipitation of the hydrotalcite support and reduction of Pd ions from the respective metal precursor salts proceed simultaneously in presence of hexamethylenetetraamine (HMTA). Decomposition of HMTA under hydrothermal conditions liberates ammonia and formaldehyde. While the precipitation of the $\mathrm{Mg}-\mathrm{Al}$ hydrotalcite is initiated by ammonia, formaldehyde effectively reduces $\mathrm{Pd}^{2+}$ to $\mathrm{Pd}^{0}$.

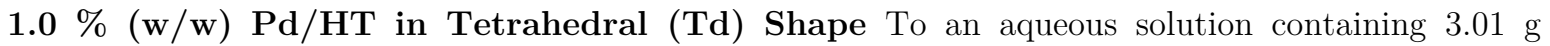
$(11.7 \mathrm{mmol})$ of $\mathrm{Mg}\left(\mathrm{NO}_{3}\right)_{2} \bullet 6 \mathrm{H}_{2} \mathrm{O}, 2.19 \mathrm{~g}(5.84 \mathrm{mmol})$ of $\mathrm{Al}\left(\mathrm{NO}_{3}\right)_{3} \bullet 9 \mathrm{H}_{2} \mathrm{O}$ and $5.76 \mathrm{~g}(41.0 \mathrm{mmol}) \mathrm{HMTA}$ in $45 \mathrm{ml}$ of de-ionized water, another aqueous solution containing $0.0157 \mathrm{~g}(0.00884 \mathrm{mmol})$ of $\mathrm{PdCl}_{2}$ and $0.01035 \mathrm{~g}(0.17 \mathrm{mmol})$ of $\mathrm{NaCl}$ in $5 \mathrm{ml}$ of de-ionized water was added and mixed together and then transferred into an autoclave. The autoclave was heated to $150{ }^{\circ} \mathrm{C}$ for $6 \mathrm{~h}$. Subsequent to natural cooling, the precipitate was filtered and washed with de-ionized water until the $\mathrm{pH}$ value of the filtrate reached 7 and finally dried overnight at $100{ }^{\circ} \mathrm{C}$.

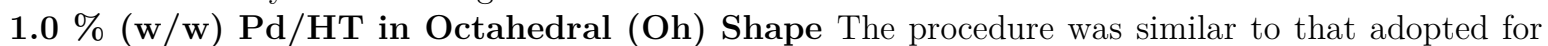
tetrahedral shaped catalyst, except that in the first solution $1.3 \mathrm{~g}(3.56 \mathrm{mmol})$ of CTAB was added along with salts of $\mathrm{Mg} \& \mathrm{Al}$ and HMTA. After the addition of second solution, the mixture was subjected to similar autoclave treatment, cooled, filtered, washed and dried at $100{ }^{\circ} \mathrm{C}$.

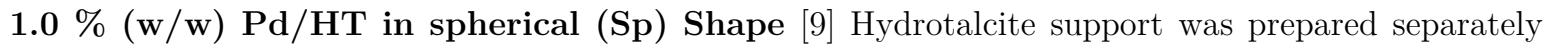
according to the method given above except adding Pd salt. A colloidal suspension of Pd nanoparticles was prepared separately by the reduction of $\mathrm{PdCl}_{2}$ with ethylene glycol in alkaline medium in presence of $\mathrm{PVP}$ as the stabilizer. Initially $\mathrm{H}_{2} \mathrm{PdCl}_{4}$ was prepared by mixing $16.5 \mathrm{mg}$ of $\mathrm{PdCl}_{2}(0.059 \mathrm{mmol} \mathrm{Pd})$ and $25 \mu \mathrm{\mu L}$ of concentrated $\mathrm{HCl}$ in a $100 \mathrm{~mL}$ glass beaker. A solution containing $25 \mathrm{mg}$ of PVP $(0.625$ $\mu \mathrm{mol}$ ) and $6 \mathrm{~mL}$ of ethylene glycol was added followed by $0.75 \mathrm{~mL}$ of $0.31 \mathrm{M} \mathrm{NaOH}$ to $\mathrm{H}_{2} \mathrm{PdCl}_{4}$ solution. The mixture was stirred for $1 / 2 \mathrm{~h}$ at $60^{\circ} \mathrm{C}$ to obtain a black colloidal suspension of Pd. To the $\mathrm{Pd}$ colloid, $0.99 \mathrm{~g}$ of hydrotalcite was added slowly and the temperature was increased to $110{ }^{\circ} \mathrm{C}$ using oil bath. The mixture was stirred continuously, monitored carefully till ethylene glycol was expelled. The catalyst was then dried in hot air oven overnight at $100{ }^{\circ} \mathrm{C}$.

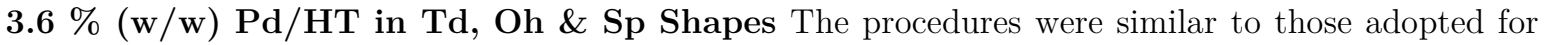
the three different shaped catalysts with $1.0 \%(\mathrm{w} / \mathrm{w}) \mathrm{Pd}$ loading, except that appropriate amounts of $\mathrm{Pd}$ salt precursors were used to achieve Pd loading of $3.6 \% \mathrm{w} / \mathrm{w}$.

\subsection{Characterization of Catalysts}

X-ray Diffraction (XRD) XRD pattern of the sample was recorded using Rigaku Miniflex (II) X-ray diffractometer using $\mathrm{Cu} \mathrm{K \alpha}$ radiation source $(\lambda=1.5401 \AA)$ with a scan rate of $3^{\circ} / \mathrm{min}$, in the $2 \theta$ range of $5-90^{\circ}$.

Transmission Electron Microscopy (TEM) TE micrographs were recorded on JEOL 3010 model $(200 \mathrm{kV})$. Few milligrams of the sample (1-2 mg) were dispersed in few $\mathrm{mL}(4-5 \mathrm{~mL})$ of ethanol by ultrasonication for 15 minutes and the sample was kept aside for $10 \mathrm{~min}$. A drop of the top layer was placed on a carbon coated copper grid and allowed to dry in air at room temperature. ImageJ software was used to calculate the crystallite sizes. Based on the mean crystallite size measured from TEM data, Pd metal dispersion was calculated using the formula (1)[10].

$$
\text { dispersion }(\%)=\frac{600 \cdot M_{P d}}{\rho_{P d} \cdot d_{n m} \cdot a_{P d} \cdot N_{a}}
$$

where, $\mathrm{M}_{\mathrm{Pd}}$ is the molecular weight of $\mathrm{Pd}(106.4 \mathrm{~g} / \mathrm{mol})$, $\mathrm{a}_{\mathrm{Pd}}$ is the atomic surface area of $\mathrm{Pd}\left(6.8 \times 10^{-20}\right.$ $\mathrm{m}^{2}$ /atom), $\rho_{\mathrm{Pd}}$ is the density of $\mathrm{Pd}\left(12.02 \mathrm{~g} / \mathrm{cm}^{3}\right), \mathrm{N}_{\mathrm{a}}$ is Avogadro's number, and $\mathrm{d}_{\mathrm{nm}}$ is the average crystallite diameter (in $\mathrm{nm}$ ) estimated from TEM data. Dispersion and the rate of hydrogenation of cinnamaldehyde (r) were used to calculate the turnover frequency, according to the formula (2)[10]. 


$$
\operatorname{TOF}\left(\mathrm{s}^{-1}\right)=\frac{r}{n_{t o t} \cdot \operatorname{dispersion}(\%)}
$$

Where $r$ is the rate of hydrogenation (moles converted per second), $n_{\text {tot }}$ is the total number of Pd moles in the reactor.

\subsection{Hydrogenation of Cinnamaldehyde}

Catalysts were evaluated for liquid phase hydrogenation of cinnamaldehyde (CAL) in a $100 \mathrm{~mL}$ Parr reactor (Model 4848) with methanol as the solvent. Reactions were conducted at $100{ }^{\circ} \mathrm{C}$, with $40 \mathrm{mg}$ of catalyst dispersed in a reaction medium containing a mixture of $13 \mathrm{~g}$ of methanol and $2.4 \mathrm{~g}$ of CAL was adopted as the standard reaction condition and hence applied to all catalysts. Prior to reaction, the reactor was first purged three times with nitrogen, followed by purging three times with $\mathrm{H}_{2}$ gas and then pressurized to 10 bar. The reaction was carried out for $1 \mathrm{~h}$ after the desired reaction temperature was reached. After each reaction, the reactor was allowed to be cooled naturally to room temperature and depressurized. The catalyst and reaction products were separated by filtration and the product stream was analyzed in PerkinElmer Clarus-500 GC equipped with FID and RTX-5MS capillary column. Hydrocinnamaldehyde (HCAL), cinnamyl alcohol (COL) and hydrocinnamyl alcohol (HCOL) were identified as the products. Since methanol was used as solvent, some amount of acetal formation was observed along with hydrogenation products. After accounting for the formation of acetal, the amount of CAL involved in hydrogenation reaction was estimated and conversion of CAL to hydrogenation products and TOF for hydrogenation was then calculated accordingly.

\section{$3 \quad$ Results and Discussions}

\subsection{Characterization of Catalysts}

X-ray diffraction patterns for $3.6 \% \mathrm{w} / \mathrm{w} \mathrm{Pd} / \mathrm{HT}$ catalysts (Fig.1) have been considered for characterization studies since d-line due to Pd (111) planes in 1\% (w/w) Pd/HT samples could not be observed clearly, because of interference by the d-line for (015) planes of HT phase. With Pd content at $3.6 \%$ (w/w) d-line due to $\mathrm{Pd}(111)$ planes at $2 \theta=40.01^{\circ}$ could be clearly seen (Fig.1 inset) for Td, Oh \& Sp shaped catalysts along with high intensity d-lines at low angles corresponding to (003) \& (006) planes of hydrotalcite phase. Intensity of (111) line is more prominent for Td shaped Pd than that for Oh \& Sp shaped Pd, since the Td phase exposes only (111) planes while Oh \& Sp phases expose both (111) as well as (100) planes [8].

Transmission electron micrographs for $1 \% \mathrm{Pd}(\mathrm{w} / \mathrm{w}) / \mathrm{HT}$ catalysts that exhibit different morphology are given below (Fig.2), along with respective histograms. The mean crystallite sizes measured are: 26.03 $\mathrm{nm}$ (Td shaped), $25.73 \mathrm{~nm}$ (Oh shaped) and $3.63 \mathrm{~nm}$ (Sp shaped). Crystallite size values obtained for $\mathrm{Td}$ and Oh shaped catalysts are close to those reported by Feng et al [8] whose method of preparation has been adopted in the present work. While the thermodynamically favoured morphology under high $\mathrm{Pd}^{2+}$ reduction rates is cubooctahedral (Spherical). With slow decomposition of HMTA to form formaldehyde as reductant, the reduction rate is slow. Hence, nano particles with tetrahedral morphology are formed during simultaneous precipitation of HT and reduction of $\mathrm{Pd}^{2+}$. However, with CTAB as capping agent, $\mathrm{Br}^{-}$ions bind preferentially to $\mathrm{Pd}$ (100) than to $\mathrm{Pd}$ (111), hence growth of (100) facets is enhanced and truncated octahedron shape with both (111) and (100) facets being observed [8]. Since the spherical shaped $1 \% \mathrm{Pd}(\mathrm{w} / \mathrm{w}) / \mathrm{HT}$ catalyst was prepared by a different method [9], the mean $\mathrm{Pd}$ crystallite size is smaller at $3.63 \mathrm{~nm}$, with its surface enclosed by mixture of facets (111) and (100) [11]. 


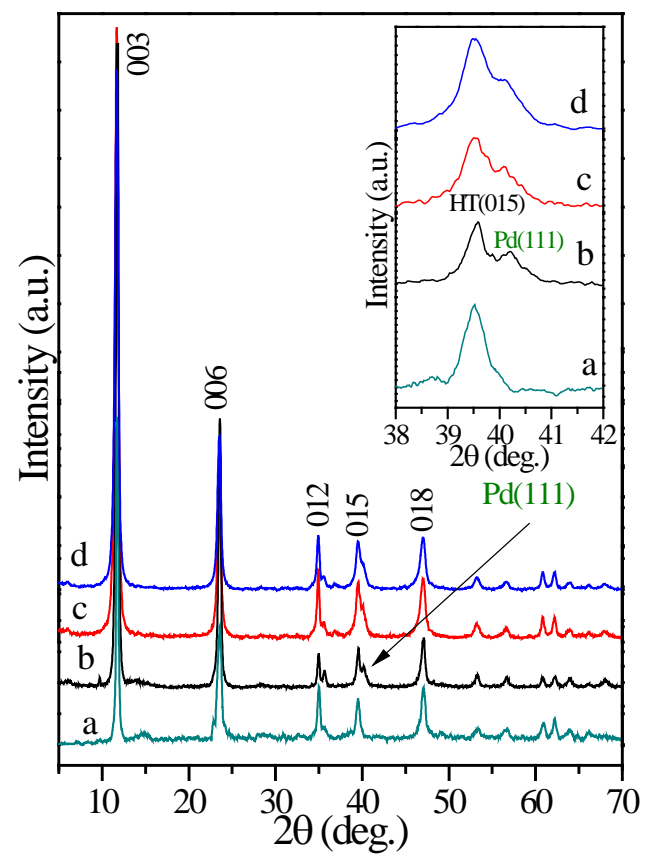

Figure 1. XRD patterns for $3.6 \%(w / w)$ Pd/HT catalysts: a) Hydrotalcite b) Tetrahedral c) Octahedral d) Spherical.
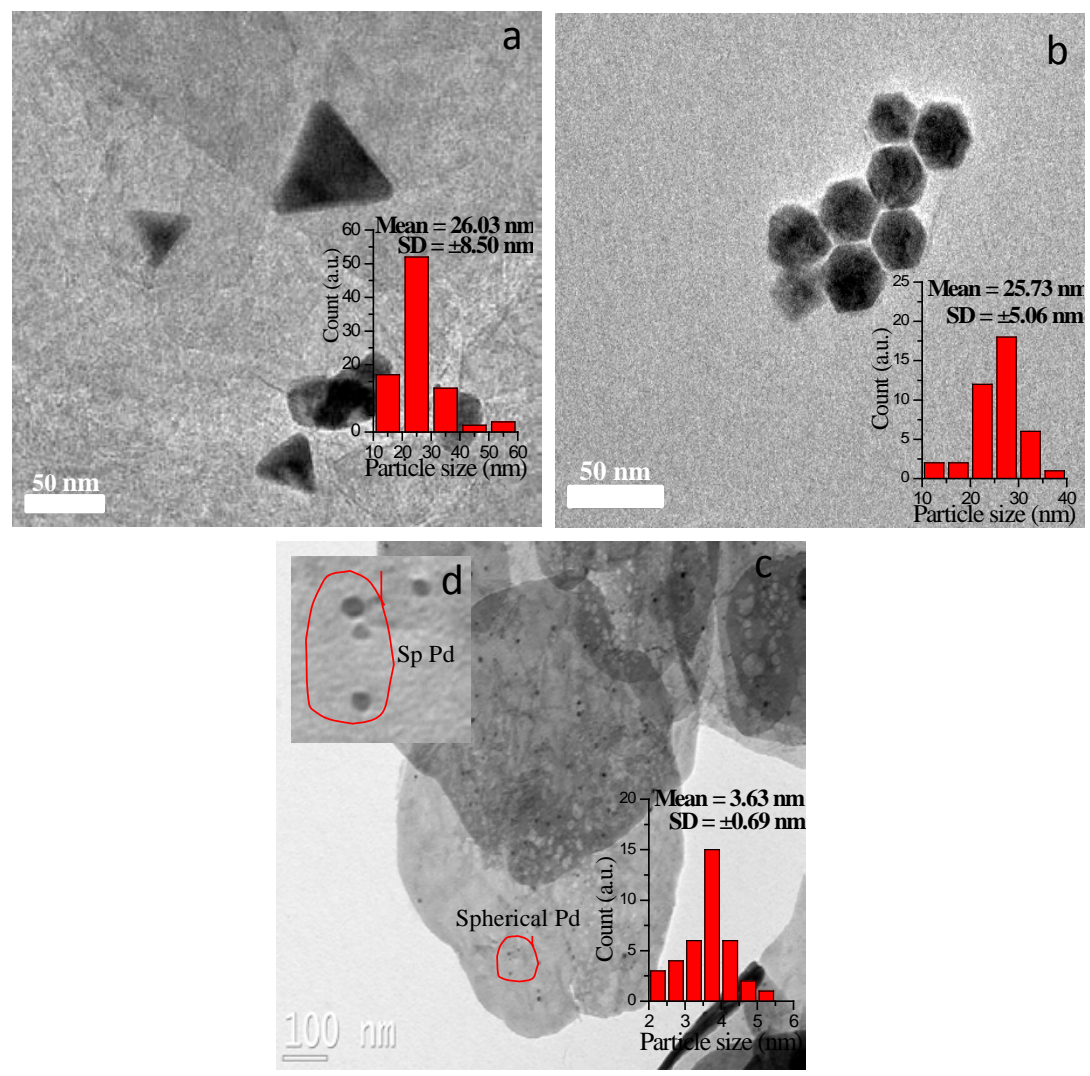

Figure 2. TE micrographs and histograms for a) Tetrahedral b) Octahedral and c) Spherical shaped Pd nano particles supported on hydrotalcite with inset d showing magnified view of Sp Pd nanoparticles. 


\subsection{Influence of Shape of Pd Nano Particles}

Based on the particle size values, Pd metal dispersion values were calculated using the formula (1). Dispersion values and CAL hydrogenation rate data were then used to calculate the turn-over frequency (TOF) values for the three catalysts according to formula (2). TOF values presented in Table.1 clearly show that $\mathrm{Td}$ shaped catalyst is more active than $\mathrm{Oh}$ and $\mathrm{Sp}$ shaped catalysts. Td shaped Pd nano particles with maximum number of exposed (111) planes, having maximum density of Pd atoms, display maximum TOF amongst the three catalysts. As Oh \& Sp shaped Pd nano particles expose both (111) and (100) planes, overall activity is less. Though the crystallite size of spherical Pd particles is small, hence the number of active sites is higher, the intrinsic activity (activity per site per unit time) is less, as the requisite active site geometry is not available. Thus, besides the crystallite size/dispersion, the shape/morphology of nano particles, that determine the active site geometry, is crucial for higher activity. Similar concept was proposed by Semagina and Kiwi-Minsker [5] who observed that the activity of nano size Pd hexagons for hydrogenation of 2-methyl-3-butyn-2-ol is less vis-à-vis nano spheres with maximum amount of exposed (111) planes. Zheng et al. [12] have demonstrated that for electro oxidation of formic acid, Pd nano cubes with exposed (100) planes are more active than Pd nano spheres of same size, due to favourable mode of adsorption of formic acid on (100) planes. It is therefore amply clear that for a specific reaction pathway, it is essential that appropriate active site geometry, as dictated by the shape/morphology of metal nano particles be made available. Invariably, synthesis and stabilization of such shape controlled nano particles is achieved by adopting suitable preparation methods.

Table 1. Activity and selectivity data for hydrogenation of cinnamaldehyde on $1 \% \mathrm{Pd}(\mathrm{w} / \mathrm{w}) / \mathrm{HT}$ Catalysts: Influence of the shape of $\mathrm{Pd}$ nano particles

\begin{tabular}{llllll}
\hline Shape & Conv. (\%) & \multicolumn{3}{l}{ Selectivity of products (\%) } & TOF $\times 10^{-2}\left(\mathrm{Sec}^{-1}\right)$ \\
\hline & & $\mathrm{HCAL}^{1}$ & $\mathrm{HCOL}^{2}$ & $\mathrm{COL}^{3}$ & \\
\cline { 3 - 5 } Tetrahedral & 25.7 & 94.2 & 0.0 & 5.7 & 7.3 \\
Octahedral & 13.9 & 98.0 & 1.9 & 0 & 3.7 \\
Spherical & 62.3 & 84.9 & 3.6 & 11.4 & 2.3 \\
\hline
\end{tabular}

Conditions: Temp. $100{ }^{\circ}$ C; Cat. wt. - $40 \mathrm{mg} ; 13 \mathrm{~g}$ methanol; $2.4 \mathrm{~g} \mathrm{CAL}^{4} ; 10$ bar $\mathrm{H}_{2}$; Reaction time $-1 \mathrm{~h}$

${ }^{1}$ Hydrocinnamaldehyde; ${ }^{2} \mathrm{Hydrociinnamyl} \mathrm{alcohol;}{ }^{3} \mathrm{Ciinnamyl}$ alcohol; ${ }^{4} \mathrm{Cinnamaldehyde}$

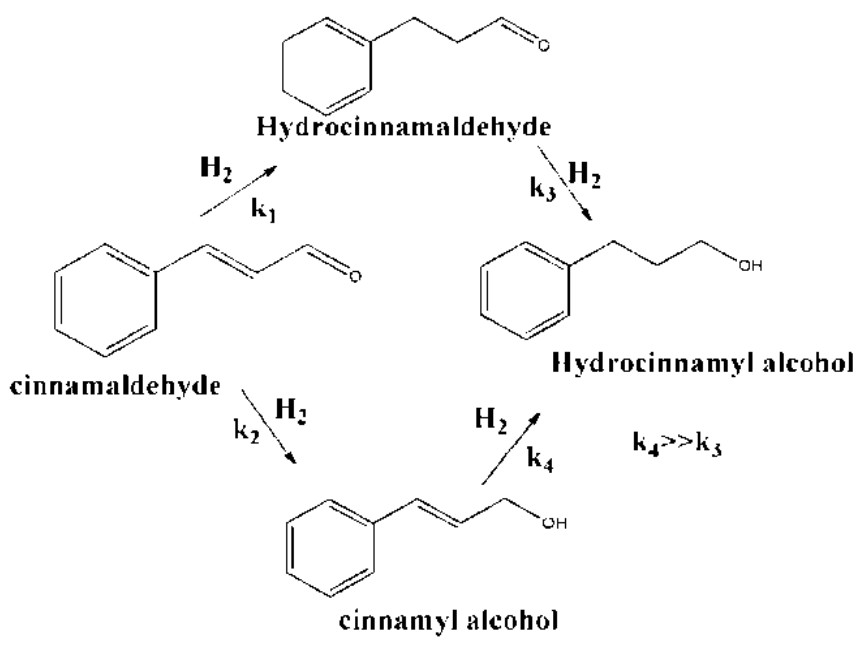

Scheme 1. Reaction pathways for hydrogenation of cinnamaldehyde.

Interestingly, both $\mathrm{Td} \& \mathrm{Oh}$ shaped Pd display very low selectivity for COL vs. 11 \% by spherical particles, indicating that mode of adsorption of $\mathrm{CAL}$ (through $\mathrm{C}=\mathrm{C}$ or $\mathrm{C}=\mathrm{O}$ bond) could perhaps dictate the selectivity towards COL. Further investigations are needed on this issue. Besides, the major product is HCAL with very low selectivity for HCOL, implying that the rate of conversion/further 
hydrogenation of HCAL to HCOL is very slow as depicted in the Scheme 1 above for hydrogenation of CAL.

\section{Conclusions}

Pd nano particles $(1 \% \mathrm{w} / \mathrm{w})$ in different shapes, i.e., tetrahedral, octahedral and spherical, supported on hydrotalcite, were prepared by simultaneous formation of hydrotalcite phase and palladium nano particles from the respective precursor salts and characterized by XRD and TEM studies. Tetrahedral shaped particles with maximum exposure of (111) planes display maximum TOF/intrinsic activity for hydrogenation of cinnamaldehyde. Octahedral and spherical shaped catalysts with the exposure of (111) and (100) planes display lower activity. Controlling morphology/shape of nano particles is crucial in order to expose appropriate crystal planes/active phase geometry suitable for the desired reaction pathway.

Acknowledgements Authors are thankful to Department of Science \& Technology, Govt. of India, New Delhi, India, for establishing research laboratories in Catalysis at NCCR and IIT Madras for all infrastructure and administrative support.

\section{References}

1. J. A. Anderson and M. Fernández Garcia, "Supported metals in Catalysis" Imperial College Press, London, 2005.

2. P. Gallezot and D. Richard, "Selective Hydrogenation of unsaturated Aldehydes" Catal. Rev.-Sci. Eng., vol. 40, no.1, pp.81-126, 1998; B. Coq and F. Figueras, "Structure-activity relationships in metals- Some aspects of particle size, bimetallic and support effects" Co-ord. Chem. Rev., vol.178-180, no.2, pp.1753-1783, 1998.

3. D .Uzio and G. Berhault "Factors governing reactivity of metallic nano particles" Catal.Rev-Sci.Eng., vol.52, no.1, pp106-121, 2010.

4. M. G. Prakash, R. Mahalakshmy, K. R. Krishnamurthy and B. Viswanathan, "Selective hydrogenation of cinnamaldehyde on nickel nano particles supported on titania-Role of catalyst preparation methods" Catal.: Sci. Technol. vol .5, no.6, pp.3313-3321, 2015.

5. N. Semagina and L. Kiwi-Minsker "Palladium Nano hexagons and Nano spheres in selective alkyne Dehydrogenation" Catal. Lett. vol.127, No.3-4, pp.334-338, 2009.

6. H. Zhang, M. Jin, Y. Xiong, B. Lim, and Y. Xia,"Shape-Controlled Synthesis of Pd nano crystals and their catalytic applications"” Acc. Chem. Res. vol. 46, no. 8, pp.1783-1794, 2013.

7. R. Wang, H. He, J. Wang, L. Liu and H. Dai "Shape-regulation: An effective way to control CO oxidation activity over noble metal catalysts" Catal. Today, vol. 201, no.1, pp. 68- 78, 2013.

8. J. Feng, X-Y Ma, Yu-Fei He, D. G. Evans, D. Li "Synthesis of hydrotalcite-supported shape-controlled Pd nano particles by a precipitation-reduction method" Appl. Catal., A, vol. 413-414, pp.10- 20, 2012.

9. Li-J. Chen, C-C. Wan, Y-Y. Wang, "Chemical preparation of Pd nanoparticles in room temperature ethylene glycol system and its application to electroless copper deposition"J. Colloid Interface Sci., vol. 297, No.2, pp. $143-150,2006$.

10. J. I. Rima, A. E. D. Holly, O. Emil, L. Leonardo, V. Philippe, A. B. Elena, "Metal-Support Interaction of Pt Nanoparticles with Ionically and Non-Ionically Conductive Supports for CO Oxidation" Electrochem. SolidState Lett. 15(3), E14-E17, 2012.

11. Y. Xiong, H. Cai, B.J. Wiley, J. Wang, M.J. Kim, Y. Xia, "Synthesis and Mechanistic Study of Palladium Nanobars and Nanorods" J. Am. Chem. Soc., 129, 3665-3675, 2007.

12. W. Zheng, J. Qu, X. Hong, K.Tedsree and S.C. E. Tsang "Probing the Size and Shape Effects of Cubic- and Spherical-Shaped Palladium Nanoparticles in the Electrooxidation of Formic Acid" ChemCatChem., vol.7, pp. $3826-3831,2015$. 